\title{
Comparative transcriptome analysis of latex from rubber tree clone CATAS8-79 and PR107 reveals new cues for the regulation of latex regeneration and duration of latex flow
}

\author{
Jinquan Chao, Yueyi Chen, Shaohua Wu and Wei-Min Tian*
}

\begin{abstract}
Background: Rubber tree (Hevea brasiliensis Muell. Arg.) is the primarily commercial source of natural rubber in the world. Latex regeneration and duration of latex flow after tapping are the two factors that determine rubber yield of rubber tree, and exhibit a huge variation between rubber tree clones CATAS8-79 and PR107.

Results: To dissect the molecular mechanism for the regulation of latex regeneration and duration of latex flow, we sequenced and comparatively analyzed latex of rubber tree clone CATAS8-79 and PR107 at transriptome level. More than 26 million clean reads were generated in each pool and 51,829 all-unigenes were totally assembled. A total of 6,726 unigenes with differential expression patterns were detected between CATAS8-79 and PR107. Functional analysis showed that genes related to mass of categories were differentially enriched between the two clones. Expression pattern of genes which were involved in latex regeneration and duration of latex flow upon successive tapping was analyzed by quantitative PCR. Several genes related to rubber biosynthesis, cellulose and lignin biosynthesis and rubber particle aggregation were differentially expressed between CATAS8-79 and PR107.

Conclusions: This is the first report about probing latex regeneration and duration of latex flow by comparative transcriptome analysis. Among all the suggested factors, it is more important that the level of endogenous jasmonates, carbohydrate metabolism, hydroxymethylglutaryl-CoA reductase (HMGR) and Hevea rubber transferase (HRT) in mevalonate (MVA) parthway for latex regeneration while the level of endogenous ethylene (ETH), lignin content of laticifer cell wall, antioxidants and glucanases for the duration of latex flow. These data will provide new cues for understanding the molecular mechanism for the regulation of latex regeneration and duration of latex flow in rubber tree.
\end{abstract}

Keywords: Hevea brasiliensis Muell. Arg, RNA-Seq, Transcriptome, Latex regeneration, Duration of latex flow

\section{Background}

Rubber tree (Hevea brasiliensis Muell. Arg.) is the main source of natural rubber [1-4]. The natural rubber is synthesized and stored in laticifer, a specific tissue densely located in the secondary phloem of trunk [5]. By successive tapping, white or yellowish milky latex is expelled and collected. The latex is the cytoplasm of laticifer cells and used to refine natural rubber. It contains numerous

\footnotetext{
* Correspondence: wmtian@163.com

Ministry of Agriculture Key Laboratory of Biology and Genetic Resources of Rubber Tree/ State Key Laboratory Breeding Base of Cultivation and Physiology for Tropical Crops, Rubber Research Institute, Chinese Academy of Tropical Agricultural Sciences, Danzhou, Hainan 571737, PR China
}

rubber particles and lutoids as well as general eukaryotic organelles [6].

Latex regeneration and duration of latex flow after tapping are important factors that determine rubber yield of rubber tree. Sucrose, water and nitrogen sources supplying from the surrounding parenchyma cells act as raw materials for latex regeneration between two tappings $[7,8]$. Isopentenyl pyrophosphate (IPP) is the direct precursor for rubber biosynthesis and mainly derived from the MVA pathway although 2-C-methyl-D-erythritol 4-phosphate (MEP) pathway is suggested to be an alternative source [6]. Catalyzing by enzymes as prenyltransferase, the rubber 
transferase, IPP initiates the subsequent extensive prenyl chain elongation process for the formation of rubber macromolecules. It is well known that tapping can promote latex regeneration and there is obvious difference in the rubber content of latex among varieties upon ethrel stimulation [7]. The duration of latex flow is influenced by various factors, such as laticifer turgor, plug formation at the end of severed laticifer, and ethrel application. Plugging of severed laticifer end leads to the termination of latex flow from the wounded site of rubber trees and has been a key limiting factor for the yield of Hevea $[9,10]$. It is widely believed that the severed laticifers are plugged by rubber coagulum as a result of rubber particle aggregation (RPA) caused by the bursting of lutoids [11,12]. Inclusions and debris of lutoids from the burst lutoids are effective in rubber particle aggregation [13,14].

With the sequence technology development, digital gene expression tag profiling recently displays huge potential for exploring biological process [15-22]. By using next-generation massively parallel sequencing technologies, Triwitayakorn et al. sequenced 2,311,497 reads from rubber tree vegetative shoot apex transcriptome, generating 23 linkage groups covering $842.9 \mathrm{cM}$ with a mean interval of $11.9 \mathrm{cM}$ per linkage group [23]. Xia et al. de novo assembled 48,768 unigenes from transcriptome data of leave and latex of rubber tree in an effort to facilitate biological, biochemical and molecular researches on rubber biosynthesis [24]. Li et al. generated 22,756 unigenes from rubber tree bark transcritome and obtained 39,257 simple sequence repeats (SSRs) markers which may be benefit for marker-assisted selection in the cross breeding program of rubber tree [25]. Mantello et al. assembled 50,384 contigs with an average length of $400 \mathrm{bp}$ from $H$. brasiliensis bark transcritome, and detected 17,927 SSRs and 404,114 single nucleotide polymorphisms (SNPs) [26]. In the present study, a comparative analysis of latex transcriptome between rubber tree clone PR107 and CATAS8-79 was performed to uncover the molecular mechanism for the regulation of latex regeneration and duration of latex flow.

\section{Results}

Difference in latex regeneration and duration of latex flow between rubber tree clone PR107 and CATAS8-79 Rubber tree clone CATAS8-79 and PR107 exhibited a huge difference in duration of latex flow (Figure 1A) and latex regeneration (Figure 1B) at each tapping. A total of about 260 $\mathrm{ml}$ of latex was gained from CATAS8-79 while only about 95 $\mathrm{ml}$ of latex from PR107 by four tappings (Figure 1B). The rubber content of latex, however, showed no difference between two clones at each tapping (Figure $1 \mathrm{C}$ ). Consequently, rubber yield of CATAS8-79 was significantly higher than that of PR107 (Figure 1D), suggesting latex regeneration in CATAS879 was more effective than that in PR107 during the interval of successive tappings.
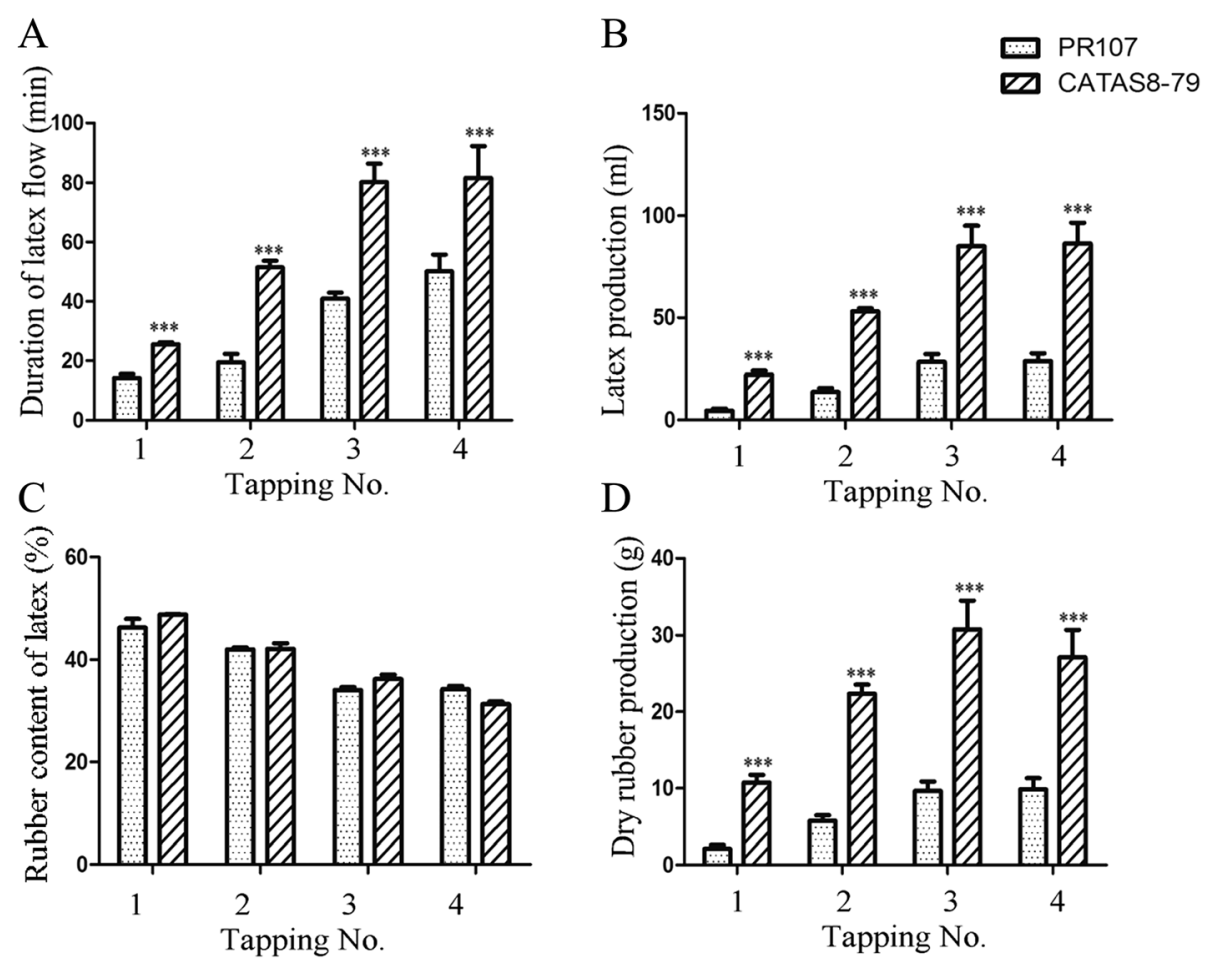

Figure 1 The difference in duration of latex flow (A), latex regeneration (B), rubber content of latex (C) and dry rubber production (D) between CATAS8-79 and PR107 upon successive tappings. Significant difference was indicated by the asterisks above the bars $\left({ }^{* * *} p<0.01\right)$. Tapping No. represented Tapping Number. 


\section{Assembling, annotation of latex transcriptome}

RNA were extracted from CATAS8-79 and PR107 at first tapping and sequenced with Illumina paired-end sequencing technology individually. After excluding low-quality reads such as empty adapters, 26 million clean reads were generated in each pool. Using SOAPdenove software, 296,736 and 308,262 contigs ranging from 100 bp to more than 3,000 bp were respectively assembled from CATAS8-79 and PR107 (NCBI accession numbers: GSE59981). By paired-end and gap-filling, contigs were further extended and finally assembled as a long sequence named "unigenes". In this way, 53,571 and 57,806 unigenes were generated from CATAS8-79 and PR107 individually. The unigenes were further integrated into 51,829 all-unigenes with an average length of $640 \mathrm{bp}$ and a N50 of 526 bp by paired-end joining (Table 1, Additional file 1: Table S1). The analyses following were carried out with all-unigenes (reffered to unigenes).

All the integrated unigenes were used to match against both the NCBI Non-redundant $(\mathrm{Nr})$ and Swissprot protein databases using BLASTx program with an E-value threshold of 1E-5. Of which, 40,373 (77.9\%) and 23,387 (45.12\%) unigenes were positively matched with $\mathrm{Nr}$ protein database and Swissprot protein database, respectively (Additional file 2: Figure S1). Clusters of Orthologous Group (COG) analysis showed that 16,242 unigenes could be divided into 25 categories (Figure 2A). Of which, "General function predicted only" represented the largest group $(2,647)$, followed by “Transcription" $(1,431)$, and "Posttranslational modification, protein turnover, chaperones" $(1,427)$. Categories of "Extracellular structures" and "Nuclear structure" only included 4 and 8 unigenes whereas 926 unigenes were classified into the category of "Carbohydrate transport and metabolism".

By comparative analysis of transcriptome data of CATAS8-79 versus PR107 under condition of FDR $\leq 0.001$ and $\mid \log 2$ Ratio $\mid \geq 1$, there were 6,726 unigenes with differential expression. Of which, 3,018 were up-regulated while 3,708 were down-regulated. "Up-regulated" means the level of gene transcripts was higher in PR107 whereas "down-regulated" means the level of gene transcripts was higher in CATAS8-79. To validate the digital gene expression (DGE), 11 unigenes were selected to amplify by qRT-PCR in the latex samples from CATAS8-79 and PR107. These unigenes were different in abundance and expression pattern on the basis of DGE data. The

Table 1 de novo assembly of $\boldsymbol{H}$. brasiliensis transcriptome

\begin{tabular}{llll}
\hline Sample & Number & N50 (bp) & Mean (bp) \\
\hline CATAS8-79 unigene & 53571 & 509 & 421 \\
PR107 unigene & 57806 & 427 & 375 \\
All unigene & 51829 & 640 & 526 \\
\hline
\end{tabular}

expression pattern of most of them by qRT-PCR was similar to the corresponding DGE data (Figure 2B).

\section{Functional analysis of the enriched categories in CATAS8-79 and PR107}

Kyoto Encyclopedia of Genes and Genomes (KEGG) analysis of all the differently expressed unigenes showed that mass of categories was differentially enriched between CATAS8-79 and PR107. Some of these differences may be related to the difference in latex regeneration and duration of latex flow between the two clones. The others may associate with the general difference in genetic background, considering that CATAS8-79 only contained one fourth descent of PR107.

Most of unigenes related to categories of stress-related proteins, cell wall biosynthesis and nitrogen requirement were significant up-regulated in PR107 (Figure 3). One unigene encoding for chitinase, eight unigenes for glucanase were detected in DGE data (Figure 4A), and unigene 49104 encoding beta-1,3-glucanase was analyzed by qRT-PCR (Figure 4B). Glutamate synthase (GS) plays a key role in integrating $\mathrm{NH}_{4}$ into amino acid, which is crucial for nitrogen requirement [27]. Recently, there is evidence that GS can directly regulate lignin biosynthesis and deposition in rice seeding [28]. Nine unigenes encoding for enzymes involving in glutamine metabolism were up-regulated in PR107 (Figure 4A), and one of them, unigene19894, was analyzed by qRT-PCR (Figure 4B). Several unigenes involving cellulose and lignin metabolism were widely activated in rubber clone PR107. Lignin is a component of cell wall and synthesized through shikimate pathway [29]. Unigenes encoding 4-coumarate-coa ligase (unigene45298, 4CL), cinnamoyl-CoA reductase (unigene13817, CCR), the key enzymes in shikimate pathway were analyzed by qRT-PCR (Figure 4B).

By contrast, most of unigenes related to categories of carbohydrate metabolism, rubber biosynthesis, hormone, and antioxidation were up-regulated in CATAS8-79 (Figure 3). Carbohydrate metabolism could provide carbon skeleton for the formation of various organic compounds [30,31]. DGE analysis showed that plenty of unigenes associating with carbohydrate metabolism were up-regulated, and the expression pattern of several unigenes mediating sucrose transportation (unigene39426 encoding sucrose transporter, SUT), starch degradation (unigene21420 encoding beta-amylase, BAM) and glycolysis (Unigene8547 encoding pyruvate kinase, PK) were analyzed by qRT-PCR (Figure 5). Both MVA and MEP pathways provide IPP, a precursor for the formation of final rubber molecule [6]. In the present study, unigenes encoding 4-hydroxy-3methylbut-2-enyl diphosphate reductase (HDR, unigene25794), 1-deoxy-D-xylulose 5-phosphate synthase (DXR, unigene3946) that is components of MEP pathway, and encoding hydroxymethylglutaryl-CoA reductase 


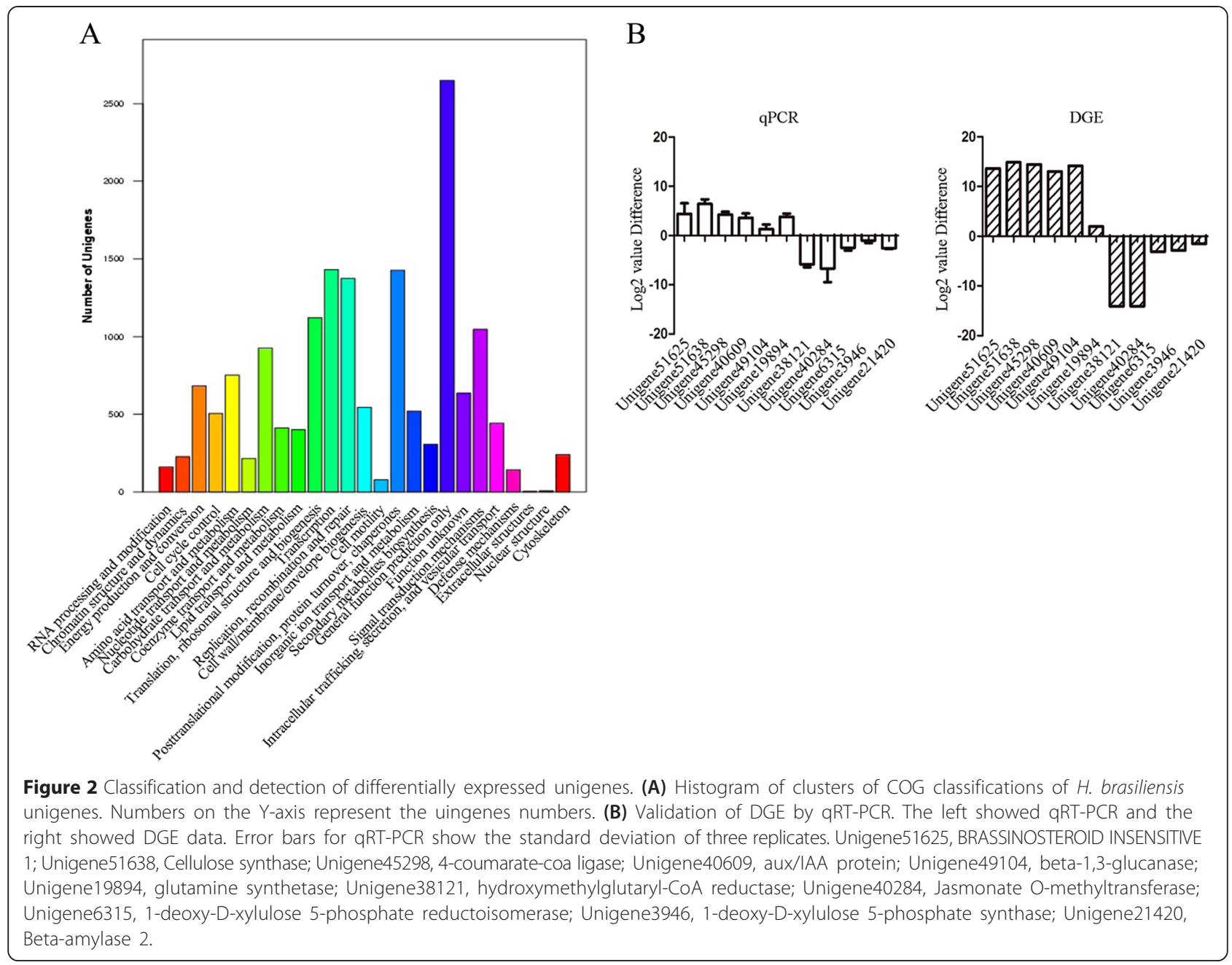

(HMGR, unigene38121) and acetyl coenzyme A acetyltransferase (AACT, unigene14484) that is the key enzymes of MVA pathway were notably up-regulated in CATAS879 (Figure 6). Of which, unigene38121 for HMGR, unigene3946 for DXR, unigene25794 for HDR were analyzed by qRT-PCR (Figure 5). Additionally, unigenes encoding farnesyl diphosphate synthase (FDPS, unigene33714), geranyl-diphosphate synthase (GPPS, unigene21852 and unigene4306), Hevea rubber transferase 2 (HRT2, Unigene37088) which directly participated in the formation of
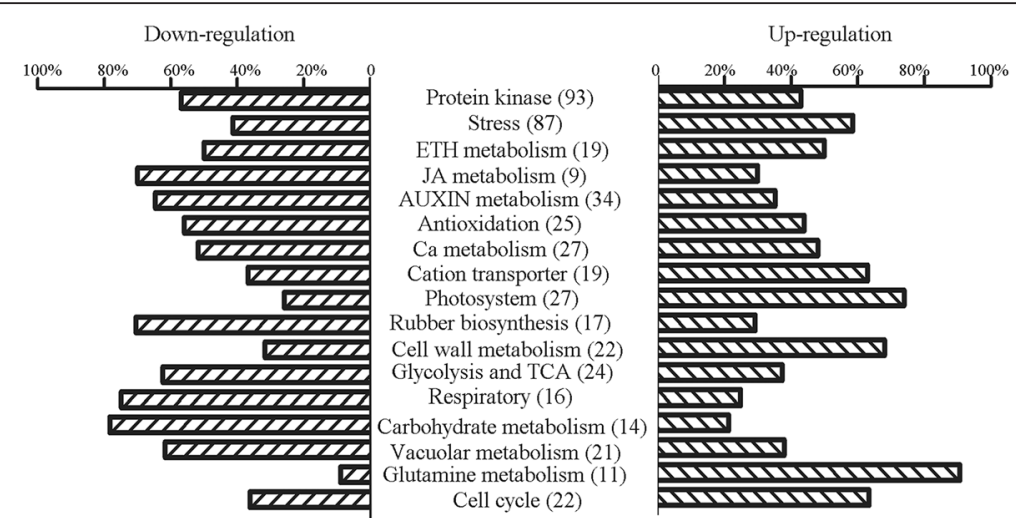

Figure 3 Histogram of metabolism categories enrichment in up-/down-regulation parts. Number in brackets showed corresponding unigenes detected in transcriptome. 


\section{A}

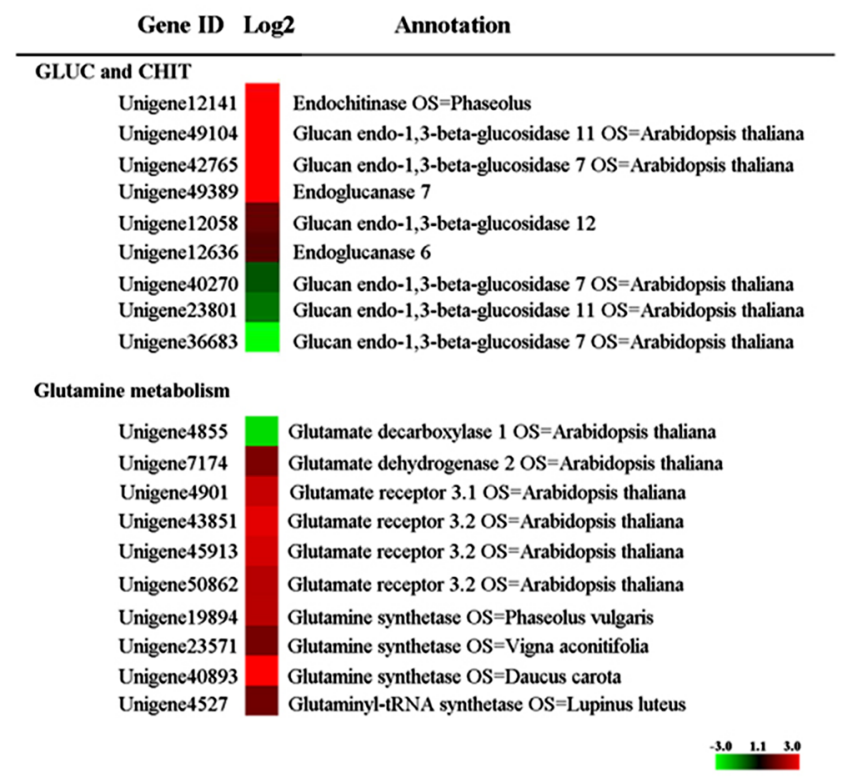

B
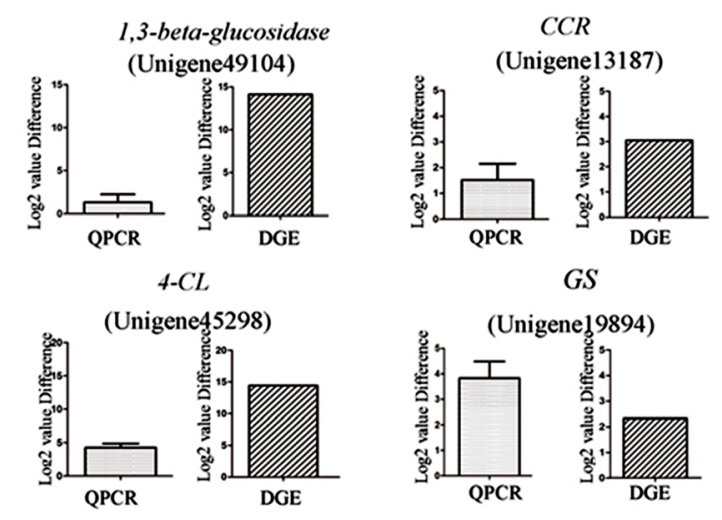

GS

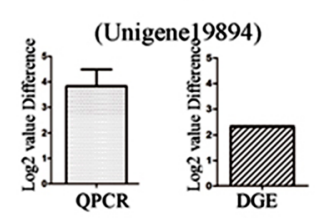

Figure 4 Differential expressed unigenes involving in cell wall biosynthesis, nitrogen metabolism and stress-related proteins. The left showed qRT-PCR (A) and the right showed heat map (B). Error bars for qRT-PCR showed the standard deviation of three replicates. DGE values displayed as heat map. Colours bar represented expression levels of each gene which were either up-regulated (red) or down-regulated (blue).

the final high-molecular weight rubber molecule were also differentially expressed (Figure 6). FDPS and HRT2related unigenes were up-regulated in CATAS8-79 whereas GPPS-related unigene was up-regulated in PR107, respectively (Figure 6). Jasmonates were pivotal to the secondary laticifer differentiation while ethylene was most effective in prolonging the duration of latex flow upon tapping in rubber tree [32,33]. In the present study, the up-regulated unigenes in JA signaling pathway in CATAS8-79 included unigene 34101 (lipoxygenase A, LOX), 38638 (lipoxygenaseA, LOX), 24544 (lipoxygenaseA, LOX), 15932 (12-oxophytodienoate reductase3, OPDR), 40284 and 39621 (jasmonate O-methyltransferase, JMT), and 38882 (Jasmonate ZIM-domain, JAZ8). And the upregulated unigenes in ethylene signaling pathway were unigene 23152 (1-aminocyclopropane-1-carboxylate oxidase homolog 1, ACO), 40400 (1-aminocyclopropane-1-carboxylate oxidase, ACO), 20082 and 17548 (ethylene-overproduction protein 1), 24000 and 20993 (ethylene-insensitive protein 2), 6206 and 17574 (EIN3-binding F-box protein 1) (Figure 6). Of which, unigene 38638 for LOX and unigene 23152 for ACO were analyzed by qRT-PCR (Figure 5). Whereas five of six unigenes (22954, 1657, 34623, 51430, 34785) encoding ethylene response factors were upregulated in PR107. In addition, antioxidants antagonized reactive oxygen species -caused membrane lipid peroxidation and had a role in keeping the integrity of lutoid [16,17]. The unigene 18915 and 15723 encoding L-ascorbate peroxidase (APX) and superoxide dismutase (SOD) were up-regulated in CATAS8-79, respectively (Figure 5).

\section{Expression pattern of unigenes related to latex regeneration and duration of latex flow upon successive tapping}

Eleven unigenes with differential expression between the two clones have been demonstrated to participate in latex regeneration or duration of latex flow. For this purpose, their expression patterns upon successive tapping were analyzed by qRT-PCR (Figure 7). Six of the eleven unigenes exhibited a big difference in their expression patterns between CATAS8-79 and PR107. Of which, unigene 49104 (beta-1,3-glucanase, HbGluc), 51638 (cellulose synthase, HbCS) and 45298 (4-coumarate-CoA ligase, $\mathrm{Hb} 4 \mathrm{CL}$ ) were up-regulated and kept significant high level in PR107 while similar expression pattern of unigene 23152 (HbACO), 38121 (HbHMGR1) and 37088 (HbHRT2) occurred in CATAS8-79 (Figure 7). The expression pattern of the other five unigenes was similar between the two clones (Figure 7).

\section{Discussion}

The rubber tree clone PR107 is an original clone selected from Wickham germplasm in 1920s. The rubber tree clone CATAS8-79 was selected from hybrid offspring of CATAS88-13 and CATAS217. The CATAS88-13 is the product of RRIM600 and PilB84 cross while the CATAS217 is selected from the cross of RRIM513 and 


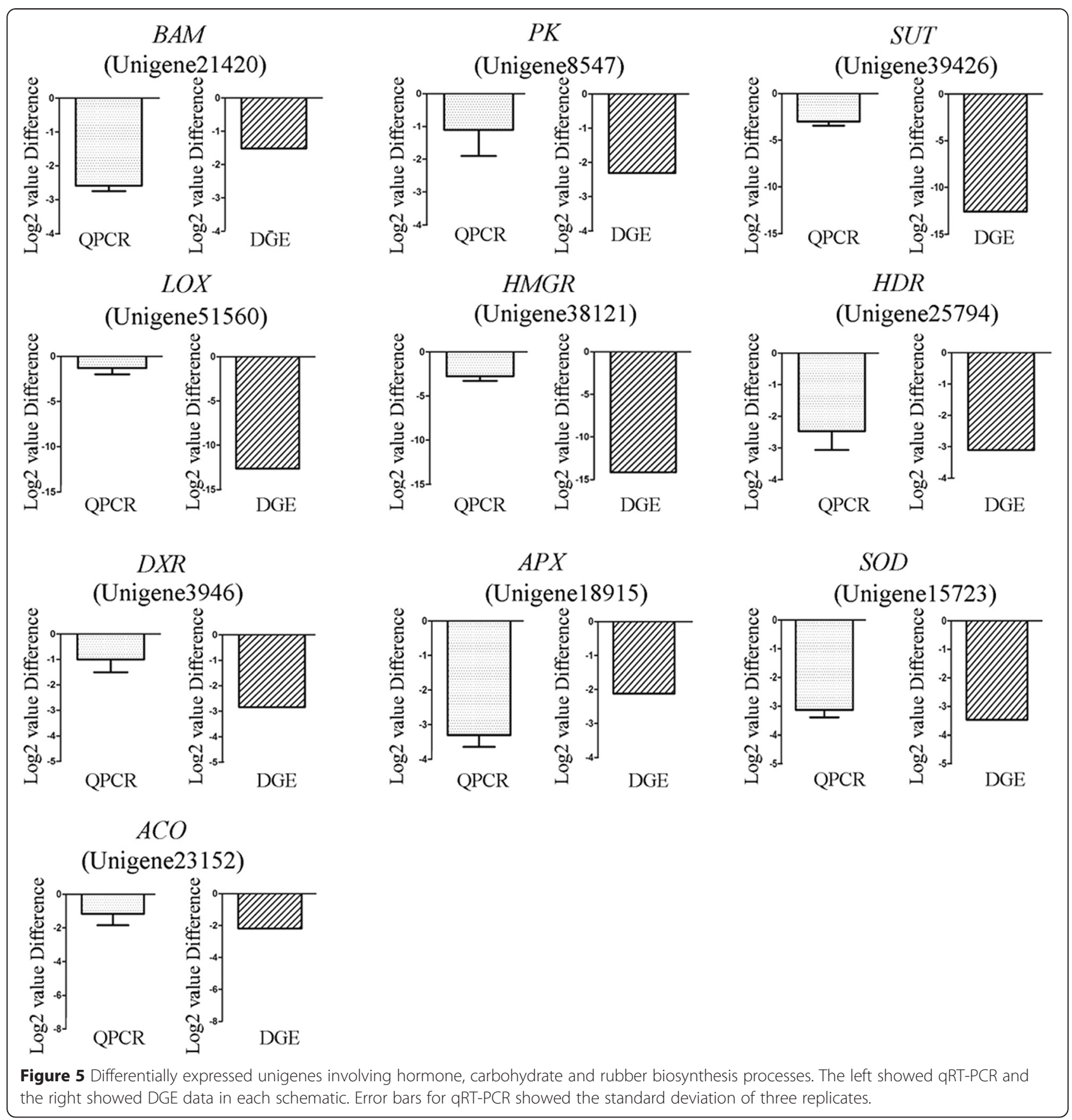

PR107. The RRIM600 are selected from the cross of Tjir1 and PB86 while RRIM513 is the hybrid offspring of PilB16 and PilA44. As there is a significant difference in duration of latex flow and latex regeneration between CATAS8-79 and PR107, analysis of the transcriptome dataset of PR107 against that of CATAS8-79 will be prospect to dissect key genes mediating the process. Some of the differentially expressed unigenes should be related to the difference in latex regeneration and duration of latex flow although the others may associate with the general difference in genetic background, considering that CATAS8-79 only contained one fourth descent of PR107.
It has long been believed that the efficiency of sucrose transportation and metabolism and rubber biosynthesis closely associates with the ability of latex regeneration between interval of successive tappings [7]. Amylase, a member of glycosyl hydrolases, is activated at weakly alkaline $\mathrm{pH}[34,35]$. One of the effects of ethrel is to enhance carbohydrate metabolism by alkalizing cytosol of laticifer cells [36], which may be ascribed to the activation of amylase. Besides, ethrel treatment can greatly up-regulate the expression of SUT genes in rubber tree [37]. In the present study, several unigenes encoding betaamylases and SUTs are found to be expressed at higher level in 


\section{Carbohydate metabolism}

\begin{tabular}{|c|c|}
\hline Gene ID & Annotation \\
\hline Unigene39426_All & Sugar transporter $\mathrm{OS}=$ Arabidopsis thaliana \\
\hline Unigene15992_All & Beta-amylase 2 OS=Arabidopsis thaliana \\
\hline Unigene21420 All & Beta-amylase 2 OS $=$ Arabidopsis thaliana \\
\hline Unigene6201_All & Sucrose synthase $O S=$ Arabidopsis thaliana \\
\hline Unigene13981_All & Sugar transporter $\mathrm{OS}=$ Arabidopsis thaliana \\
\hline Unigene20545 All & Sucrose synthase OS=Phaseolus aureus \\
\hline Unigene12965_All & Sucrose synthase $O S=$ Glycine $\max$ \\
\hline Unigene781_All & Beta-amylase $1 O S=$ Arabidopsis thaliana \\
\hline Unigene29858_All & Sucrose synthase $O S=$ Glycine max \\
\hline Unigene21244_All & 1,4-alpha-glucan branching enzyme $O S=$ Arabidopsis \\
\hline Unigene3404_All & Beta-amylase 1 OS $=$ Oryza sativa \\
\hline \multirow{4}{*}{$\begin{array}{l}\text { Unigene11346_All } \\
\text { Unigene41888_All } \\
\text { Unigene49183_All }\end{array}$} & Beta-amylase $8 \mathrm{OS}=$ Arabidopsis thaliana \\
\hline & Neutral invertase $O S=$ Arabidopsis thaliana \\
\hline & Starch branching enzyme OS=Solanum tuberosum \\
\hline & -3.01 .13 .0 \\
\hline
\end{tabular}

\section{Rubber biosynthesis}

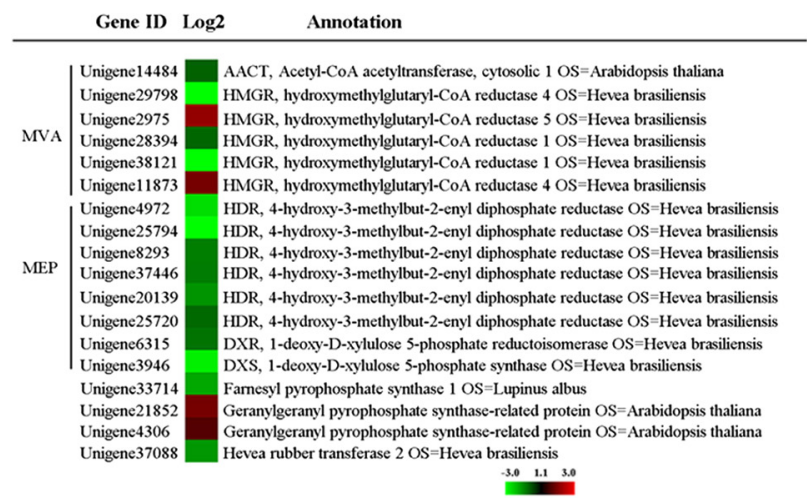

Hormone metabolism

\begin{tabular}{|c|c|c|}
\hline & Gene ID & Annotation \\
\hline JA metabo & & \\
\hline & Unigene34101 & Lipoxygenase A OS=Solanum lycopersicum \\
\hline Lox & Unigene38638 & Lipoxygenase $1 \mathrm{OS}=$ Solanum tuberosum \\
\hline & Unigene 24544 & Lipoxygenase A OS=Solanum lycopersicum \\
\hline & Unigene 46229 & Lipoxygenase 2, chloroplastic $\mathrm{OS}=$ Arabidopsis thaliana \\
\hline & Unigene 46018 & Allene oxide cyclase 3 , chloroplastic $O S=$ Arabidopsis thaliana \\
\hline & Unigene45784 & Allene oxide synthase, chloroplastic $O S=$ Linum usitatissimum \\
\hline & Unigene15932 & 12-oxophytodienoate reductase $3 \mathrm{OS}=$ Solanum lycopersicum \\
\hline JMT & $\begin{array}{l}\text { Unigene } 40284 \\
\text { Unigene39621 }\end{array}$ & $\begin{array}{l}\text { Jasmonate } \mathrm{O} \text {-methyltransferase } \mathrm{OS}=\text { Brassica rapa subsp. pekinensis } \\
\text { Jasmonate } \mathrm{O} \text {-methyltransferase } \mathrm{OS}=\text { Arabidopsis thaliana }\end{array}$ \\
\hline & Unigene38882 & JAZ8 OS $=$ Arabidopsis thaliana 7 \\
\hline ETH metal & olism & \\
\hline & Unigene3225 & 1-aminocyclopropane-1-carboxylate oxidase homolog $1 \mathrm{OS}=$ Arabidopsis thaliana \\
\hline ACO & Unigene23152 & 1-aminocyclopropane-1-carboxylate oxidase homolog $1 O S=$ Arabidopsis thaliana \\
\hline & Unigene 40400 & 1-aminocyclopropane-1-carboxylate oxidase OS=Dictyostelium mucoroides \\
\hline & Unigene20082 & Ethylene-overproduction protein $1 O S=$ Arabidopsis thaliana \\
\hline & Unigene17548 & Ethylene-overproduction protein $1 \mathrm{OS}=$ Arabidopsis thaliana \\
\hline & Unigene25812 & Ethylene-overproduction protein $1 O S=$ Arabidopsis thaliana \\
\hline & Unigene 24000 & Ethylene-insensitive protein $2 O S=$ Arabidopsis thaliana \\
\hline & Unigene20993 & Ethylene-insensitive protein $2 O S=$ Arabidopsis thaliana \\
\hline & Unigene22954 & Ethylene-responsive transcription factor RAP2-3 OS $=$ Arabidopsis thaliana \\
\hline & Unigene1657 & Ethylene-responsive transcription factor $\mathrm{ERF} 118 \mathrm{OS}=$ Arabidopsis thaliana \\
\hline ERF & Unigene34623 & Ethylene-responsive transcription factor WRI 1 OS $=$ Arabidopsis thaliana \\
\hline & Unigene51430 & Ethylene-responsive transcription factor WRI 1 OS $=$ Arabidopsis thaliana \\
\hline & Unigene34785 & Ethylene-responsive transcription factor $3 \mathrm{OS}=\mathrm{Nicotiana}$ tabacum \\
\hline & Unigene39286 & Ethylene-responsive transcription factor $12 \mathrm{OS}=$ Arabidopsis thaliana \\
\hline & Unigene 46844 & ENN3-binding F-box protein $2 \mathrm{OS}=$ Arabidopsis thaliana \\
\hline & Unigene6206 & ENN3-binding F-box protein $1 O S=$ Arabidopsis thaliana \\
\hline & Unigene16574 & ENN3-binding F-box protein $1 O S=$ Arabidopsis thaliana \\
\hline & Unigene51436 & Ethylene receptor OS=Prunus persica \\
\hline
\end{tabular}

Figure 6 Heat map of genes involve in carbohydrate metabolism, rubber biosynthesis and hormone metabolism. DGE values displayed as heat map. Colours bar represented expression levels of each gene which were either up-regulated (red) or down-regulated (blue).

CATAS8-79 than that in PR107, suggesting a more efficiency of sucrose transportation and carbohydrate metabolism occurred in CATAS8-79 (Figure 6). IPP is biosynthesized though both MVA and MEP pathway and runs several enzymatic reactions to form the final high-molecular weight rubber molecule [7]. In present study, five unigenes encoding for HMGR and six unigenes encoding for HDR are detected with differently expression between two clones. Further sequence analysis shows four HDR-like members (unigene 4972, 25794, 8293, 37446) are perfectly blasted to one $H b H D R$ with a Genbank number EU881977 whereas five HMGR-like unigenes are matched to three HbHMGR genes (unigene11873, unigene29798 for HbHMGR4, unigene28394, unigene38121 for HbHMGR1, unigene2975 for HbHMGR5) respectively. Previous work shows that the transcripts of HbHMGR1 are most abundant in latex [38]. The present study shows that HMGR1-like unigenes are significantly up-regulated in CATAS8-79 while HbHDR displays a similar pattern between the two clones, suggesting HMGR1 in MVA pathway is critical for providing IPP.

Post-IPP processes include initiation and elongation of rubber macromolecules. FDPS family catalyzes the biosynthesis of farnesyl diphosphate (FDP). The FDP acts as the prime which is essential for initiating prenyl chain whereas HRT family is crucial for integrating IPP units into prenyl chain $[39,40]$. Two members of HRT family (HRT1 and HRT2) are reported in rubber tree [41]. In vitro analysis shows that only HRT2 has rubber transferase activity and may play a key role in extending prenyl chain [41]. In this study, unigenes related to HRT2 and FDPS are found to be expressed at higher level in CATAS8-79 than that in PR107, indicating that HRT2 and FDPS are crucial for enhancing rubber initiation and elongation. The higher expression level of HRT2 and FDPS like unigenes is in line with the higher rubber yield per tapping (Figure 1).

Jasmonate signaling plays a pivotal role in activating the secondary laticifer differentiation and activating the biosynthesis of secondary metabolites [42-45]. Although several members of COI1-JAZ-MCY module have been characterized in rubber tree and JA signaling is suggested to have an important role in regulating rubber biosynthesis in laticifer cells [46-48], the difference in the level of endogenous JAs in laticifer cells among Hevea germplasm remains largely unknown. The present study suggests that the level of endogenous JAs may be higher in the laticifer cells of CATAS 8-79 than that in the laticifer cells of PR107, considering that the expression of unigenes encoding enzymes such as LOX, OPDR, JMT is significantly up-regulated in CATAS8-79. 


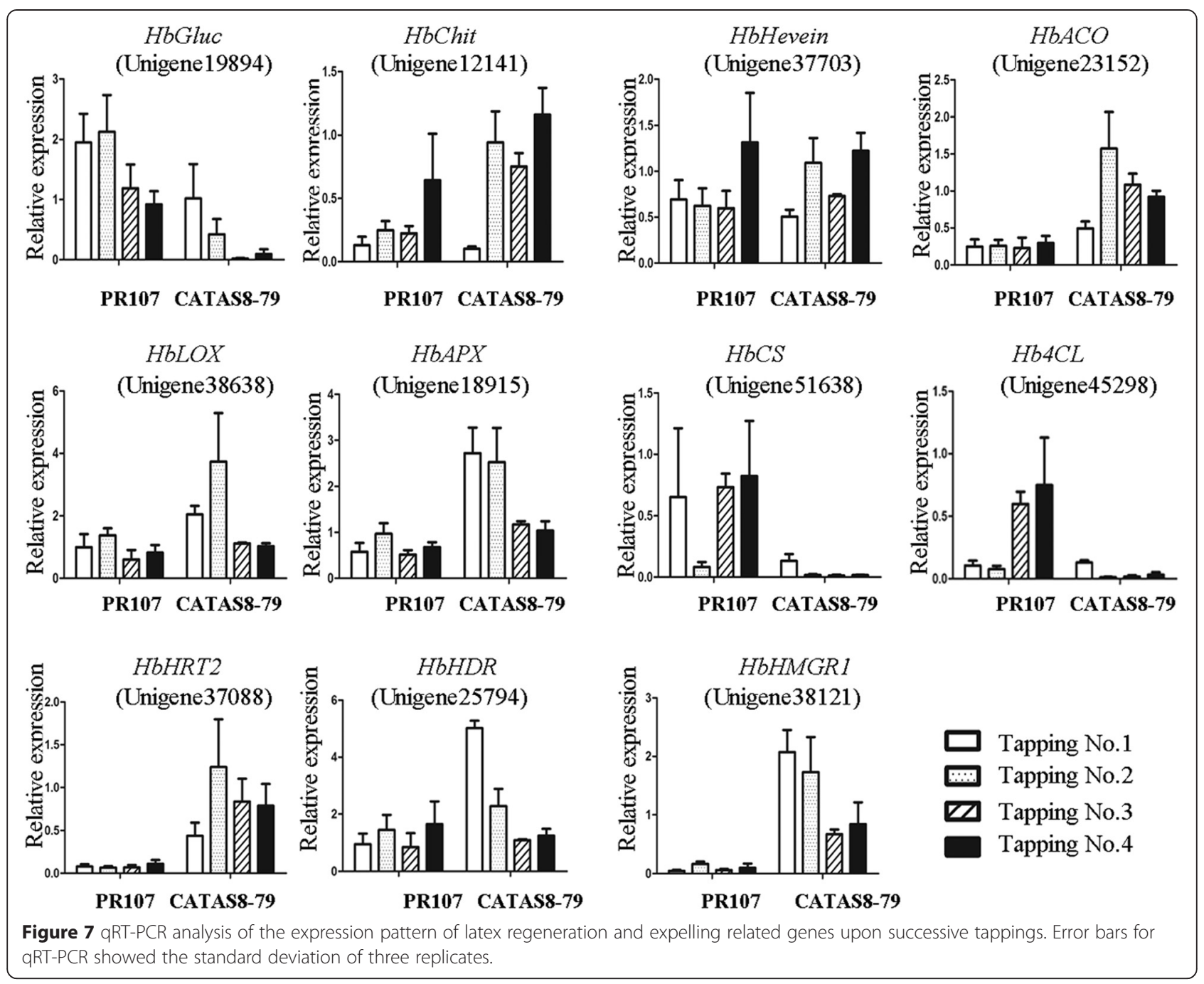

Ethrel, an ethylene releaser, is very effective in prolonging duration of latex flow [32]. One of the explanations for ethrel-induced prolongation of latex flow is its effect on maintaining the turgor pressure of laticifer and surrounding liber cells via its differential regulation on the aquaporins on the plasma membrane and tonoplast [49]. Considering that unigenes such as ACO, Ethylene overproduction protein and EIN3-binding F-box protein are up-regulated in CATAS8-79, the activity of ethylene biosynthesis and signal transduction in the laticifer cells of CATAS 8-79 may be higher than that of PR107. Besides, such factors as laticifer turgor, antioxidants, inclusions and debris of lutoid, and proteins in C-serum are suggested to influence the duration of latex flow [10-12]. After tapping, the bursting of lutoid particles leads to the release of hevein, chitinase, and glucanase. These protein inclusions are effective in rubber particle aggregation [5]. In the present study, it is the unigene encoding for glucanase other than the unigenes encoding for hevein and chitinase that is significantly up-regulated in PR107, suggesting that glucanase is more important in inducing rubber particle aggregation. The turgor pressure of laticifer in CATAS8-78 is significantly higher than that in PR107 [50]. In the present study, the unigenes enconding for enzymes (HbCS; Hb4CL) of cellulose and lignin biosynthesis are highly activated in RP107. This suggests that difference in turgor pressure may primarily associate with the difference in lignin content of laticifer cell wall between the two clones.

\section{Conclusions}

Taken together, comparative transcriptome analysis reveals new cues at molecular level for the difference in duration of latex flow and latex regeneration between rubber tree clone CATAS8-79 and PR107 (Figure 8). Up-regulated expression of unigenes $H b L O X$ and $H b O P D R$ in the pathway of JA biosynthesis, $H b S U T, H b B A M$ and $H b P K$ mediating carbohydrate metabolism, $H b H M G R 1$ in MVA parthway and $H b H R \mathrm{~T} 2$ directly in rubber biosynthesis is important for enhanced latex regeneration. It may 


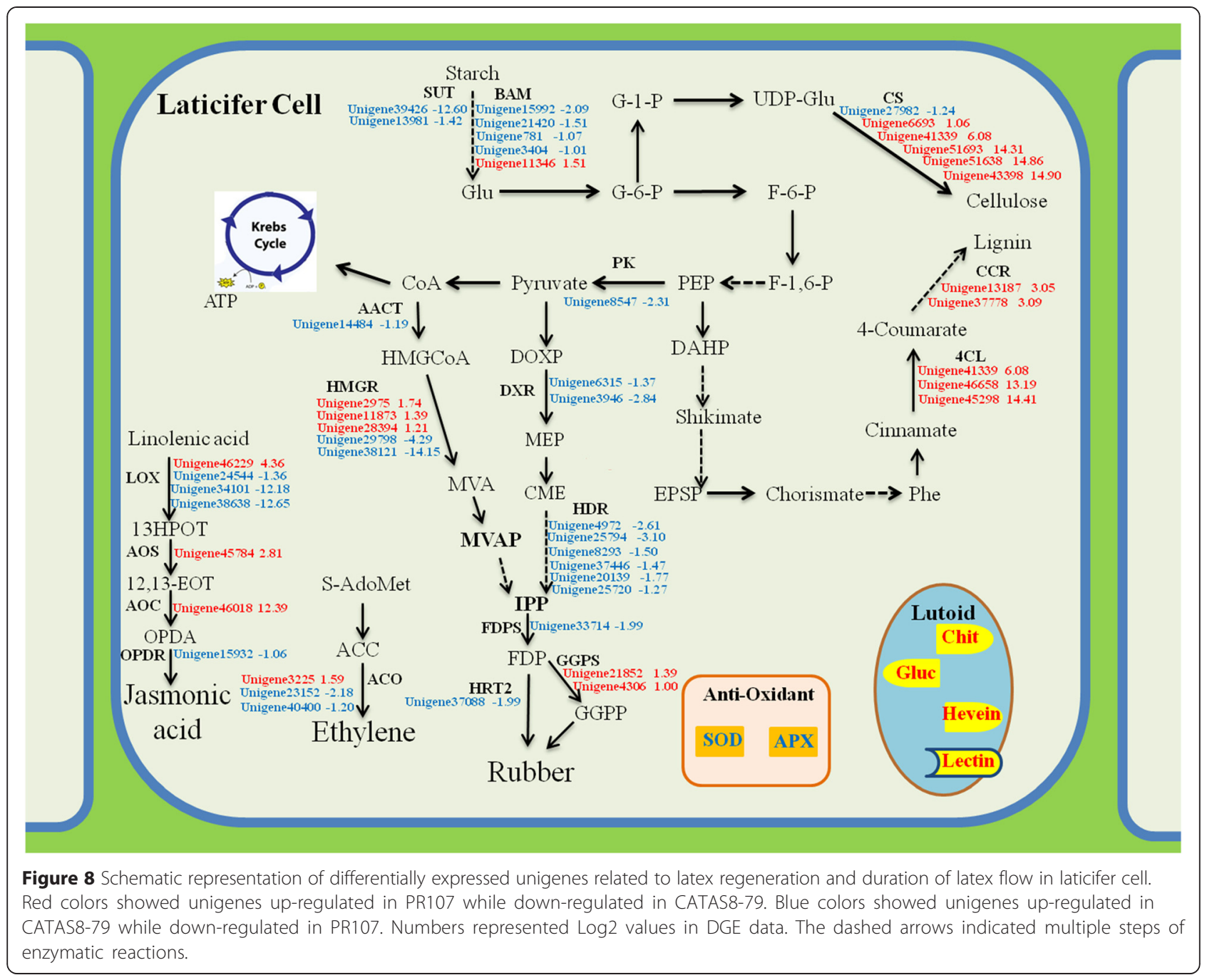

be essential for prolonging duration of latex flow that upregulated expression of unigenes $\mathrm{HbACO}$ mediating ethylene biosynthesis, $H b A P X$ and $H b S O D$ for antagonizing reactive oxygen species, but down-regulated expression of unigenes $\mathrm{Hb} 4 \mathrm{CL}$ and $\mathrm{HbCCR}$ in the pathway of lignin biosynthesis and HbGluc for rubber particle aggregation.

\section{Methods}

\section{Plant materials}

Seven-year-old virgin trees of rubber tree clone CATAS8-79 and PR107 were grown at the Experimental Station of the Rubber Research Institute of the Chinese Academy of Tropical Agricultural Sciences in Danzhou city, Hainan province, P.R. China. The virgin trees with same circumference were seleced in this study. For RNA-Seq, latex from five individual trees by the first tapping was pooled for each clone. The samples were immediately stored at $-80^{\circ} \mathrm{C}$ until RNA extraction. For real time-PCR and determination of physiological parameters, latex was individually collected from another batch of five trees for each clone upon the first, second, third and forth tapping, respectively. All the selected virgin trees were tapped with a tapping system of $S / 2, d / 2$ (a half spiral pattern, every two days) at 6:00 am in August, 2013.

\section{Rubber content determination}

To determine rubber content of latex, $100 \mu$ l of acetic acid were dropped into $1 \mathrm{~g}$ of fresh latex to obtain rubber coagula. The sample rubber coagula were washed in water for $2 \mathrm{~h}$, then dried overnight at $55^{\circ} \mathrm{C}$ and weighted. The experiments were repeated three times.

\section{RNA isolation and sequencing}

Total latex RNA was extracted as described [7] and RNA integrity was evaluated by NanoDrop (Thermo Scientific Inc., USA). The double strand cDNA was synthesized using SuperScript ${ }^{\circ}$ Double-Stranded cDNA Synthesis Kit (Invitrogen Inc., USA), and purified and added single nucleotide A (adenine) to the end with QiaQuick PCR extraction kit. Finally, sequencing adaptors were ligated to the cDNA fragments. The required fragments were 
purified by $2 \%$ agarose gel electrophoresis and enriched by PCR amplification. The library products were sequenced via Illumina HiSeq 2000 by Beijing Genomics Institute (Shenzhen, China). The original image datasets was transferred into sequence datasets by base calling. Clean reads were obtained by removing adaptor sequence, low quality sequences, empty tags, low complexity, and tags with only one copy.

\section{Transcriptome de nove assembly, annotation and classification}

Transcriptome de novo assembly was carried out using a de Bruijn graph and the SOAPdenovo as previously described [26]. Under a certain overlap length $(k-m e r=$ 29), SOAPdenovo combined overlapping reads into contigs. Adjacent contigs were constructed into scaffolds by read mate pairs. Within the scaffold, the connected contigs used ' $\mathrm{N}$ ' to represent unknown sequences and insert size information. Finally, paired-end information was used to fill the gap of scaffolds to obtain the extended sequences with fewer Ns, which were defined as unigenes for further analysis.

All unigenes were used for BLAST searches (E-value $<1 \mathrm{E}-5$ ) against databases as NCBI $\mathrm{Nr}$ (http:// www.ncbi.nlm.nih.gov/), Swissprot (http://www.expasy.ch/sprot/), KEGG (http://www.genome.jp/kegg/) and COG (http://www.ncbi.nlm.nih.gov/cog/). The best aligning results were chosen for unigene annotation. The aligning results were selected with an order of $\mathrm{Nr}$, Swiss-Prot, KEGG and COG.

To classify the unigenes, the Blast2GO program was used to get GO annotation based on molecular function, biological process and cellular component. All unigenes were also aligned to the COG database to predict possible functions and KEGG pathway database to perform pathway assignments.

\section{Digital gene expression analysis}

A rigorous algorithm was developed to identify differentially expressed genes between two different DGE libraries (CATAS8-79 versus PR107). Raw clean tags in each library were normalized to Tags Per Million (TPM) to obtain normalized gene expression level. Differential digital gene expression was deemed with FDR value $\leq 0.001$ and $\mid \log 2$ Ratio $\mid \geq 1$ in sequence counts across libraries. "Up-regulated" means the level of gene transcripts were higher in PR107 whereas "down-regulated" means the level of gene transcripts were higher in CATAS8-79.

\section{Quantitative PCR analysis}

Approximately $1 \mu \mathrm{g}$ of RNA was used for reverse transcription based on the introduction of RevertAid ${ }^{\text {tm }}$ First Strand cDNA Synthesis Kit (Thermo Scientific Inc., USA). qPCR was performed on the CFX96 System (Bio-Rad
Laboratories Inc., USA) with SYBR PrimeScript RT-PCR Kit (TaKaRa Biotechnology, Japan). Nine housekeeping genes (Hb18s, HbActin, HbELF1A, HbRH2B, HbRH8, HbYLS8, HbUBC2A, HbUBC2B, HbUBC4) were selected to evaluate the stability by the software package NormFinder (version 0.953, http://www.mdl.dk/publicationsnormfinder.htm) [51]. $H b U B C 2 B$ and $H b U B C 4$ were suitable for reference genes in the present study due to its stability in latex samples upon tapping (Additional file 3: Figure S2). Expression values were normalized for differences in cDNA input using parallel reactions employing primers designed against a reference gene $H b U B C 2 B$ (HQ323247). All primer pairs used in this article were list in as an Additional file 4: Table S2.

\section{Statistical analyses}

The statistical analyses were ANOVAs carried out on raw data. A $t$ test (Student's $t$ test) was carried out for the significance analysis. ${ }^{* * * *}$ indicates very significantly difference $(\mathrm{p}<0.01)$.

\section{Availability of supporting data}

The authors confirm that all data underlying the findings are fully available without restriction. Data are available at the following URL: http://www.ncbi.nlm.nih.gov/geo/ query/acc.cgi?acc=GSE59981.

\section{Additional files}

Additional file 1: Table S1. Statistics of DGE sequencing from CATAS8-79 and PR107 libraries.

Additional file 2: Figure S1. Species distribution of unigenes with matches in $\mathrm{Nr}(\mathrm{A})$ and Swissport (B) databases. The species distribution is shown as a total homologous sequences in the NCBI Nr (A) and Swissport (B) databases with an E-value $<10-5$.

Additional file 3: Figure S2. Average expression stability values of the candidate reference genes evaluated by NormFinder. The least stable genes are on the left, and the two most stable genes are on the right.

Additional file 4: Table S2. Primers used in this paper.

\section{Abbreviations}

4CL: 4-coumarate-coa ligase; ACO: 1-aminocyclopropane-1-carboxylate oxidase; AOS: Allene oxide synthase; AOC: Allene oxide cycalse; BAM: Beta-amylase; CCR: Cinnamoyl-CoA reductase; Chit: Chitinase; COG: Clusters of Orthologous Group; CS: Cellulose synthase; DXR: 1-deoxy-D-xylulose 5-phosphate reductoisomerase; DGE: Digital gene expression; FDPS: Farnesyl diphosphate synthase; Gluc: Glucanase; GO: Gene Ontology; GPPS: Geranyl-diphosphate synthase; GS: Glutamate synthase; HDR: 4-hydroxy-3methylbut-2-enyl diphosphate reductase; HMGR: Hydroxymethylglutaryl-CoA reductase; HRT: Hevea rubber transferase; IPP: Isopentenyl diphosphate; JA: Jasmonic acid; JMT: Jasmonate O-methyltransferase; KEGG: Kyoto Encyclopedia of Genes and Genomes; LOX: Lipoxygenase; MEP: 2-C-methyl-Derythritol 4-phosphate; MVA: Mevalonate; OPDR: 12-oxophytodienoate reductase; PK: Pyruvate kinase; APX: L-ascorbate peroxidase; qRT-PCR: Quantitative reverse transcription-PCR; RPA: Rubber particle aggregation; SNP: Single nucleotide polymorphism; SOD: Superoxide dismutase; SUT: Sucrose transporter.

\section{Competing interests}

The authors declare that they have no competing interests. 


\section{Authors' contributions}

Conceived and designed the experiments: WMT. Performed the experiments: JC, YC and SW. Analyzed the data: JC and SW. Wrote the paper: JC and WMT. All authors read and approved the final manuscript.

\section{Acknowledgements}

This work was supported by the National Natural Science Fundation of China (31170642) and the Special Program for Key Basic Research of the Ministry of Science and Technology, China (2012CB723005).

\section{Received: 31 December 2014 Accepted: 2 April 2015 \\ Published online: 18 April 2015}

\section{References}

1. Mooibroek H, Cornish K. Alternative sources of natural rubber. Appl Microbiol Biotechnol. 2000;53:355-65.

2. Hagel JM, Yeung EC, Facchini PJ. Got milk? The secret life of laticifers. Trends Plant Sci. 2008;13:631-9.

3. Rahman AY, Usharraj AO, Misra BB, Thottathil GP, Jayasekaran K, Feng Y, et al. Draft genome sequence of the rubber tree Hevea brasiliensis. BMC Genomics. 2013;14:75.

4. van Beilen JB, Poirier Y. Establishment of new crops for the production of natural rubber. Trends Biotechnol. 2007;25:522-9.

5. Wang X, Shi M, Wang D, Chen Y, Cai F, Zhang S, et al. Comparative proteomics of primary and secondary lutoids reveals that chitinase and glucanase play a crucial combined role in rubber particle aggregation in Hevea brasiliensis. J Proteome Res. 2013;12:5146-59.

6. Chow KS, Mat-Isa MN, Bahari A, Ghazali AK, Alias H, Mohd-Zainuddin Z, et al Metabolic routes affecting rubber biosynthesis in Hevea brasiliensis latex. J Exp Bot. 2012;63:1863-71.

7. Tang C, Huang D, Yang J, Liu S, Sakr S, Li H, et al. The sucrose transporter HbSUT3 plays an active role in sucrose loading to laticifer and rubber productivity in exploited trees of Hevea brasiliensis (para rubber tree). Plant Cell Environ. 2010;33:1708-20.

8. Tian WM, Zhang H, Yang SG, Shi MJ, Wang XC, Dai LJ, et al. Molecular and biochemical characterization of a cyanogenic $\beta$-glucosidase in the inner bark tissues of rubber tree (Hevea brasiliensis Muell. Arg.). J Plant Physiol. 2013;170:723-30

9. d'Auzac J, Jacob JL, Chrestin H. Physiology of rubber tree latex. Florida: CRC Press; 1989. p. 314

10. Kongsawadworakul $\mathrm{P}$, Chrestin H. Laser diffraction: a new tool for identification and studies of physiological effectors involved in aggregation-coagulation of the rubber particles from Hevea latex. Plant Cell Physiol. 2003;44:707-17.

11. Wititsuwannakul $R$, Pasitkul $P$, Jewtragoon $P$, Wititsuwannakul D. Hevea latex lectin binding protein in C-serum as an anti-latex coagulating factor and its role in a proposed new model for latex coagulation. Phytochemistry. 2008;69:656-62.

12. Salomez M, Subileau M, Intapun J, Bonfils F, Sainte-Beuve J, Vaysse L, et al. Micro-organisms in latex and natural rubber coagula of Hevea brasiliensis and their impact on rubber composition, structure and properties. J App Microbiol. 2014;117:921-9.

13. Wahler D, Gronover CS, Richter C, Foucu F, Twyman RM, Moerschbacher $\mathrm{BM}$, et al. Polyphenoloxidase silencing affects latex coagulation in Taraxacum species. Plant Physiol. 2009;151:334-46.

14. Leclercq J, Martin F, Sanier C, Clément-Vidal A, Fabre D, Oliver G, et al. Over-expression of a cytosolic isoform of the $\mathrm{HbCuZnSOD}$ gene in Hevea brasiliensis changes its response to a water deficit. Plant Mol Biol. 2012;80:255-72.

15. Wang Z, Fang B, Chen J, Zhang X, Luo Z, Huang L, et al. De novo assembly and characterization of root transcriptome using Illumina paired-end sequencing and development of cSSR markers in sweet potato (Ipomoea batatas). BMC Genomics. 2010;11:726.

16. Zenoni S, Ferrarini A, Giacomelli E, Xumerle L, Fasoli M, Malerba G, et al. Characterization of transcriptional complexity during berry development in Vitis vinifera using RNA-Seq. Plant Physiol. 2010;152:1787-95.

17. Zhang G, Guo G, Hu X, Zhang Y, Li Q, Li R, et al. Deep RNA sequencing at single base-pair resolution reveals high complexity of the rice transcriptome. Genome Res. 2010:20:646-54

18. Garg R, Patel RK, Tyagi AK, Jain M. De novo assembly of chickpea transcriptome using short reads for gene discovery and marker identification. DNA Res. 2011;18:53-63.
19. Iorizzo M, Senalik DA, Grzebelus D, Bowman M, Cavagnaro PF, Matvienko M, et al. De novo assembly and characterization of the carrot transcriptome reveals novel genes, new markers, and genetic diversity. BMC Genomics. 2011;12:389.

20. Shi CY, Yang $H$, Wei $C L$, Yu O, Zhang ZZ, Jiang CJ, et al. Deep sequencing of the Camellia sinensis transcriptome revealed candidate genes for major metabolic pathways of tea-specific compounds. BMC Genomics. 2011;12:131.

21. Wong MM, Cannon $\mathrm{CH}$, Wickneswari R. Identification of lignin genes and regulatory sequences involved in secondary cell wall formation in Acacia auriculiformis and Acacia mangium via de novo transcriptome sequencing. BMC Genomics. 2011;12:342.

22. Chow KS, Ghazali AK, Hoh CC, Mohd-Zainuddin Z. RNA sequencing read depth requirement for optimal transcriptome coverage in Hevea brasiliensis. BMC Res Notes. 2014;7:69.

23. Triwitayakorn K, Chatkulkawin P, Kanjanawattanawong S, Sraphet S, Yoocha T, Sangsrakru D, et al. Transcriptome sequencing of Heved brasiliensis for development of microsatellite markers and construction of a genetic linkage map. DNA Res. 2011:18:471-82.

24. Xia ZH, Xu HM, Zhai JL, Li DJ, He CZ, Huang X. RNA-Seq analysis and de novo transcriptome assembly of Hevea brasiliensis. Plant Mol Biol. 2011;77:299-308

25. Li D, Deng Z, Qin B, Liu X, Men Z. De novo assembly and characterization of bark transcriptome using Illumina sequencing and development of ESTSSR markers in rubber tree (Hevea brasiliensis Muell. Arg.). BMC Genomics. 2012;13:192

26. Mantello CC, Cardoso-Silva CB, da Silva CC, de Souza LM, Scaloppi Junior EJ, de Souza GP, et al. De novo assembly and transcriptome analysis of the rubber tree (Hevea brasiliensis) and SNP markers development for rubber biosynthesis pathways. PLoS One. 2014;9:e102665.

27. Pujade-Renaud V, Clement A, Perrot-Rechenmann C, Prevot JC, Chrestin H, Jacob $J$, et al. Ethylene-induced increase in glutamine synthetase activity and mRNA levels in Hevea brasiliensis latex cells. Plant Physiol. 1994:105:127-32.

28. Ohashi M, Ishiyama K, Kusano M, Fukushima A, Kojima S, Hanada A, et al. Lack of cytosolic glutamine synthetase 1;2 in vascular tissues of axillary buds causes severe reduction in their outgrowth and disorder of metabolic balance in rice seedlings. Plant J. 2015;81:347-56.

29. Tzin V, Galili G. New insights into the shikimate and aromatic amino acids biosynthesis pathways in plants. Mol Plant. 2010;3:956-72.

30. Bolton MD. Primary metabolism and plant defense-fuel for the fire. Mol Plant-Microbe Interact. 2009:22:487-97.

31. Dieuaide-Noubhani M, Alonso AP, Rolin D, Eisenreich W, Raymond P. Metabolic flux analysis: recent advances in carbon metabolism in plants. Plant Syst Biol. 2007:97:213-43.

32. Zhu J, Zhang Z. Ethylene stimulation of latex production in Hevea brasiliensis. Plant Signal Behav. 2009;4:1072-4.

33. Hao BZ, Wu JL. Laticifer differentiation in Hevea brasiliensis: induction by exogenous jasmonic acid and linolenic acid. Ann Bot. 2000;85:37-43.

34. Monroe JD, Storm AR, Badley EM, Lehman MD, Platt SM, Saunders LK, et al. $\beta$-amylase 1 and $\beta$-amylase 3 are plastidic starch hydrolases in Arabidopsis that seem to be adapted for different thermal, $\mathrm{pH}$, and stress conditions. Plant Physiol. 2014;166:1748-63.

35. Li J, Zhao H, Huang W. Mechanism of proanthocyanidins-induced alcoholic fermentation enhancement in Saccharomyces cerevisiae. J Ind Microbio Biotechnol. 2014:41:1793-802.

36. Amalou Z, Bangratz J, Chrestin H. Ethrel (ethylene releaser)-induced increases in the adenylate pool and transtonoplast $\Delta \mathrm{pH}$ within Hevea latex cells. Plant Physiol. 1992;98:1270-6.

37. Dusotoit-Coucaud A, Kongsawadworakul P, Maurousset L, Viboonjun U, Brunel N, Pujade-Renaud V, et al. Ethylene stimulation of latex yield depends on the expression of a sucrose transporter (HbSUT1B) in rubber tree (Hevea brasiliensis). Tree Physiol. 2010;30:1586-98.

38. Sando T, Takaoka C, Mukai Y, Yamashita A, Hattori M, Ogasawara N, et al. Cloning and characterization of mevalonate pathway genes in a natural rubber producing plant, Hevea brasiliensis. Biosci Biotechnol Biochem. 2008;72:2049-60.

39. Koyama T. Molecular analysis of prenyl chain elongating enzymes. Biosci Biotechnol Biochem. 1999:63:1671-6.

40. Tangpakdee J, Tanaka Y, Ogura K, Koyama T, Wititsuwannakul R, Wititsuwannakul D, et al. Isopentenyl diphosphate isomerase and prenyl 
transferase activities in bottom fraction and C-serum from Hevea brasiliensis. Phytochemistry. 1997:45:261-7.

41. Ogura K, Koyama T. Enzymatic aspects of isoprenoid chain elongation. Chem Rev. 1998;98:1263-76.

42. Asawatreratanakul K, Zhang YW, Wititsuwannakul D, Wititsuwannakul R, Takahashi S, Rattanapittayaporn A, et al. Molecular cloning, expression and characterization of CDNA encoding cis-prenyltransferases from Hevea brasiliensis. A key factor participating in natural rubber biosynthesis. Eur J Biochem. 2003;270:4671-80.

43. Shitan N, Sugiyama A, Yazaki K. Functional analysis of jasmonic acid-responsive secondary metabolite transporters. Methods Mol Biol. 2013;1011:241-50.

44. Misra RC, Maiti P, Chanotiya CS, Shanker K, Ghosh S. Methyl jasmonate-elicited transcriptional responses and pentacyclic triterpene biosynthesis in sweet basil. Plant Physiol. 2014;164:1028-44.

45. Goldhaber-Pasillas GD, Mustafa NR, Verpoorte R. Jasmonic acid effect on the fatty acid and terpenoid indole alkaloid accumulation in cell suspension cultures of Catharanthus roseus. Molecules. 2014;19:10242-60.

46. Peng S-Q, Xu J, Li H-L, Tian W-M. Cloning and molecular characterization of HbCOl1 from Hevea brasiliensis. Biosci Biotechnol Biochem. 2009;73:665-70.

47. Tian W-M, Huang W-F, Zhao Y. Cloning and characterization of HbJAZ1 from the laticifer cells in rubber tree (Hevea brasiliensis Muell. Arg.). Trees. 2010;24:771-9.

48. Pirrello J, Leclercq J, Dessailly F, Rio M, Piyatrakul P, Kuswanhadi K, et al. Transcriptional and post-transcriptional regulation of the jasmonate signalling pathway in response to abiotic and harvesting stress in Hevea brasiliensis. BMC Plant Biol. 2014;14:341.

49. Tungngoen K, Kongsawadworakul P, Viboonjun U, Katsuhara M, Brunel N, Sakr S, et al. Involvement of HbPIP2;1 and HbTIP1;1 aquaporins in ethylene stimulation of latex yield through regulation of water exchanges between inner liber and latex cells in Hevea brasiliensis. Plant Physiol. 2009;151:843-56.

50. An F, Cahill D, Rookes J, Lin WF, Kong LX. Real-time measurement of phloem turgor pressure in Hevea brasiliensis with a modified cell pressure probe. Bot Stud. 2014;55:19

51. Li H, Qin Y, Xiao X, Tang C. Screening of valid reference genes for real-time RT-PCR data normalization in Hevea brasiliensis and expression validation of a sucrose transporter gene HbSUT3. Plant Sci. 2011;181:132-9.

\section{Submit your next manuscript to BioMed Central and take full advantage of:}

- Convenient online submission

- Thorough peer review

- No space constraints or color figure charges

- Immediate publication on acceptance

- Inclusion in PubMed, CAS, Scopus and Google Scholar

- Research which is freely available for redistribution 